\title{
Optimization of the combined explosion hardening processes
}

\author{
Viktor Shchetynin ${ }^{1}$, Roman Arhat ${ }^{1}$, Vladimir Drahobetskyi ${ }^{1}$, Vyacheslav Puzyr $^{1}$, and Dmitriy Maloshtan ${ }^{1}$ \\ ${ }^{1}$ Kremenchuk Mykhailo Ostrohradskyi National University, 20 Pershotravneva Str., Kremenchuk, 39600, Ukraine
}

\begin{abstract}
The proposed method for calculating the loading parameters makes it possible to determine the wear parameters after explosion hardening. The calculation method is simple and less time consuming compared with calculation methods that involve the use of nonlinear programming methods. The main methods of increasing the wear resistance of mining equipment parts using explosion methods are generalized. The reserve for increasing the wear resistance consists in the optimization of deformation parameters during the power and thermal intensification of processes and the development of new methods and technologies of hardening. The factors (parameters) of the studied processes: explosive cladding, alloying, hardening, are formulated. Optimization of the processes under consideration is possible by decomposing the process into simpler ones with subsequent optimization of the parameters of these processes and the synthesis of the obtained solutions. For the first time, a solution to the multicriteria problem of two-stage explosion hardening is presented. It is proposed to split the process into simpler ones. Optimization criteria are proposed for each of the simplified processes. The problem is reduced to a conditional extremum problem, which is solved by composing the Lagrange function. By transforming the wear equation, the optimal ratio of strength and ductility for parts operating under abrasive wear conditions is determined.
\end{abstract}

\section{Introduction}

This research examines the processes of increasing the wear resistance of mining equipment parts using pulsed energy sources. These processes include explosive cladding with wear-resistant materials, explosion hardening, explosion-thermal hardening and frictional hardening.

The research is devoted to solving the topical scientific and technical problem of increasing the resource and operational reliability of parts and assemblies of mining equipment. This is due to the fact that at present the physical wear and tear of production assets of the mining and metallurgical complex (MMC) of Ukraine is $70-75 \%$. The costs of repair, installation and dismantling of parts of equipment operating under intensive wear conditions are comparable with its high cost. Nevertheless, MMC occupies a major place in the national economy of Ukraine, providing a third of industrial production and more than $40 \%$ of export profits. The most effective and cheap methods for increasing the wear resistance and durability of mining equipment parts for buckets and bucket teeth for excavators, hammer crusher bits, ball and rod mill armor linings are methods of impulse hardening, alloying and cladding.

The reserve for increasing the wear resistance of mining equipment parts is optimization of parameters, power and thermal intensification of these processes, development of fundamentally new methods and technologies of hardening $[1,2]$. In addition, the search for technologies for restoring the wear resistance of parts without dismantling them is also promising. Therefore, the purpose of this research consists in developing a universal analytical method for calculating the optimal parameters of the processes of increasing the wear resistance and durability of parts using pulsed energy sources.

Optimization of the problems of impulse metalworking is quite fully covered in the literature [3-8]. Most of the optimization problems of deformation and shape changing are reduced to determining the extreme values of the response parameters and the values of the corresponding determining parameters $[9,10]$. The method of choosing the geometry of the deforming tool according to the criterion of the minimum surface load in the areas of contact with the workpiece is of interest [3].

In this case, the system of regularities of elastic-plastic deformation underlying the theory of rolling are replaced by variational principles. In this case, the problem is reduced to the calculus of variations and optimal control and can be studied in two ways. Either the extreme trajectories (Euler-Lagrange theory), or by experimental trajectories (Hamilton-Jacobi theory) could be determined. The task is reduced to finding «geodesic lines» which the shortest arcs connecting two are given points on a certain surface. This minimal problem is a typical example of a questions vast class for the calculus of variations.

In general, in most research, it is believed that obtaining an extreme value of a parameter is possible only for certain values of all factors, which significantly complicates the calculations and obtaining the final results. A number of researchers $[11,12]$ take into account the factors that are most significant, using the method of

\footnotetext{
*Corresponding author: shchetinin23@gmail.com
} 
frozen parameters or successive approximations. To determine the significance of the factors, the values of several of them are fixed while others change. Then the values of the latter, minimizing the full power of forming, are determined $[13,14]$.

A number of practical problems of the metal processing theory have an alternative nature. For example, reducing material consumption increases the likelihood of stamping scrap. The solution of such problems requires the use of the mathematical apparatus of probability theory.

The design of technological processes is usually reduced to solving a multivariate problem with full or partial formalization of the compared options optimality criterion. The cost of a product could be a fully formalized, complex criterion. However, the widespread use of the cost criterion in the design of sheet-stamping production processes is difficult both due to the lack of the necessary information support for the dependence of the cost of products on the technology of its production, and on performance indicators.

It is more expedient to evaluate the efficiency of a technological process as a physical system using the state function, i.e. entropy. It is characterized by the tendency of systems to an equilibrium state, equilibrium and to equiprobable microscopic chaotic molecular processes. At the same time, plastic deformation occurs under conditions of inhomogeneity not only of the stress-strain state, but also of the structure, chemical potential, and temperature. I.e., shaping and hardening processing are characterized by a complex thermomechanical and physicochemical environment. As a result, irreversible energy flows arise, causing local entropy production.

The process of plastic deformation and the subsequent depletion of the parts resource obtained by pressure treatment methods is ultimately determined by the ratio of competing flows. This is an energy flow that seeks to destroy the metal during processing and operation, or prevents it. The predominant flow determines the reliability of the structure at this operation stage. However for problem solving, it is necessary to have a system of equations for the kinetic state of a physical object and dynamic return. At the same time, the solution and composition of these equations are considered only for some special cases without obtaining quantitative indicators.

In addition, the development of the mathematical apparatus, mathematical modeling methods and computer technology significantly increases the reliability and calculations quality. Therefore, according to a number of researches [15] experimental and static methods for studying the processes of manufacturing and operation of machines structural elements are giving way to a less costly method of mathematical modeling. Regarding the modeling of technological processes of forming parts, this really takes place [16]. The calculation technique [16] for assessing the parameters of the state of parts during manufacturing, repair and subsequent operation is the methodological basis for the development of the theory and practice of creating highly reliable structures, assemblies and parts. A set of mathematical models of the service parameters of parts evolution during operation: the accumulation of damage and a decrease in fatigue life, etc. do not solve the issues of the need for preventive maintenance and measurements of parts and structures as a whole; nevertheless, the methods of «operations research» taking into account static data allow solving a complex of problems of economics, operation and repair. However, the general strategic task of uniformity of machine parts service life is solved by the joint efforts of constructors, technologists, operators, mathematicians and economists.

However, the determination of the significance of all factors is compatible in terms of labor intensity with the procedure for enumerating all the parameters.

The foregoing allows us to state that to date, in the theory of sheet stamping production, considerable scientific knowledge has been accumulated in the use of methods of the optimal control theory and mathematical modeling to increase the efficiency of metal forming processes. The widespread use of optimal control methods for solving specific technological problems is relevant.

In conclusion of the paragraph, it should be noted that a variety of problems of the optimal control theory is inherent in the process of sheet-stamping production, and the fact that the application of the methods of this theory to shaping technologies is in it's development stage.

\section{Material and methods}

For a functional description of the processes under consideration, it is necessary to formulate the factors (parameters) of the researched processes. The main parameters of explosion welding (kinematic and physical) include: speed of movement of the point of contact $v_{c}$, dynamic impact angle $\beta$, plate driving speed $v_{0}$, pressure, impact duration, temperature in the impact zone. In addition, the process efficiency is largely determined by the technological parameters:

- detonation velocity $D$, which characterizes the type of explosive charge;

- dimensionless welding parameter $r$ - the ratio of the mass of the explosive to the mass of the driver (plate, pipe);

- distance between the welded workpieces - welding gap $h$.

The factors affecting the degree of explosive hardening include the applied pressure, the duration of the impact pulse, the nature of the loading, and the velocity of the linear pulse.

For explosive friction hardening the factors are the same as for explosion hardening, only instead of the linear impulse velocity the velocity of the friction material is used.

In addition, the physical and mechanical characteristics of the processed material affect the efficiency of the explosion welding process and the degree of hardening.

Optimization of the analyzed processes by theoretical analysis of numerical modeling is possible using the calculation method described in [17, 18]. The latter includes a set of models that characterize the properties of the original blanks and the evolution of these properties 
during processing and operation. Taking into account the fact that the researched processes depend on a smaller number of factors, we adapt the method used in structural mechanics by V.Z. Vlasov and in the processing of metals by pressure by V.I. Ershov, applying them to the processes of explosion welding and hardening by a laidon charge of an explosive, converging shock waves, explosive-thermal, explosive-frictional, multiple combined explosion, multiple compression by reflected shock waves.

\section{Calculation}

The processes of explosion welding, hardening by converging shock waves, a laid-on charge of explosives, proceed under the action of one type of external load, the rest of the processes occur under the action of several independent or dependent loads.

In the former case the process is described by function $p_{1}\left(y_{1} \ldots y_{k}\right)$ :

$$
F_{i}=F_{i}\left[\begin{array}{l}
p_{1}\left(y_{1}, y_{2}, \ldots, y_{k}\right) ; \varepsilon_{1}, \varepsilon_{2}, \ldots, \varepsilon_{l} ; \\
z_{1}, z_{2}, \ldots, z_{m} ; S_{1}, S_{2}, \ldots, S_{n} ; \ldots
\end{array}\right] .
$$

In the latter case:

$$
F_{i}=F_{i}\left[\begin{array}{l}
p_{1}\left(p_{1}, p_{2}, \ldots, p_{k}\right), p_{2}\left(p_{1}, p_{3}, \ldots, p_{n}\right), \ldots, \\
p_{n}\left(p_{1}, p_{2}, \ldots, p_{n-1}\right) ; \varepsilon_{1}, \varepsilon_{2}, \ldots, \varepsilon_{l} \\
z_{1}, z_{2}, \ldots, z_{m} ; S_{1}, S_{2}, \ldots, S_{n} ; \ldots
\end{array}\right]
$$

The processes under consideration are fast.

Therefore, practically without error, function (2) can be replaced by functions of the form:

$$
F_{i} \approx F_{i}^{\prime}=F_{q}\left(F_{1}, F_{2}, \ldots, F_{n}\right),
$$

where $F_{i}$ - parameter characterizing the efficiency of the considered process;

$p_{1}, p_{2}, \ldots, p_{k}$ - factors that characterize the influence of each type of load on the process of cladding with a wearresistant layer, hardening by the previously listed methods of explosion hardening;

$\varepsilon_{1}, \varepsilon_{2}, \ldots, \varepsilon_{l}-$ factors characterizing the conditions of deformation;

$z_{1}, z_{2}, \ldots, z_{m}$ - factors characterizing the properties of the deformable workpiece (geometric and physicalmechanical);

$S_{1}, S_{2}, \ldots, S_{n} \quad-\quad$ factors describing structural transformations in the hardened material;

$F_{i}^{\prime}$ - the function approximating function $F_{i}$;

$F_{q}$ - determines the influence of a complex of interactions of factors, $p_{k}$ on $F_{i}$.

From the specific conditions of deformation hardening, functions $F_{1}, F_{2}, \ldots, F_{n}$ are determined by one of the types of load.

When the process takes place under the influence of several independent external loads $n>1$ function $F_{q}$ must be determined from additional conditions. To determine function $F_{q}$ for the processes under consideration, the equality of the intensity of deformations is extremely uniform [17] at the minimum total power of deformation.

It should be noted that for fast processes of explosive hardening, frictional and shock-wave loading of the hardened material acts for several microseconds. Subsequent loading by a technological equipment element occurs much later and lasts several tens of milliseconds, so these processes can be considered as processes occurring under the action of two or more independent external loads.

\section{Results}

Let us consider the practical use of the analyzed method for the process of explosion-friction hardening. The steps are as follows:

1) the process is subdivided into two simple processes: explosive loading and frictional interaction of the technological friction sliding pad with the workpiece. Explosive loading by a plane shock wave of the padworkpiece system results in the occurrence of a stressed triaxial uneven compression and driving of the technological pad. Contact friction stresses act on the contact surface of the workpiece with the tool. They have a significant effect on the final deformable state of the workpiece;

2) determining a functional link $F_{i}=F_{i}\left(p_{k}\right)$;

3 ) in accordance with equation (2), the functions $F_{q}$ are determined. In this case it is considered that $p_{1}, p_{2}$ are not independent, but may influence each other. Here $p_{1}$ affects the speed of movement of the technological pad and the force of normal pressure between the pad and the workpiece.

4) determining the relation between factors $p_{1}, p_{2}$ from the condition of the minimum deformation energy when the deformations of the hardened surface are limited to the limit uniform one.

At the final stage we obtain the explosive-friction process dependence on many factors.

A method based on the decomposition of a combined process into simple ones and subsequent synthesis makes it possible to optimize the processes under consideration. Process conditions, properties of the deformable workpiece and structural changes in the surface layer of the workpiece. In this case:

$$
F_{i}=F_{i}\left(p_{1}, p_{2}\right) \text {. }
$$

We expand function $F_{i}$ in Taylor's series at points ( $p_{l}$, $0)$ and $\left(0, p_{2}\right)$ and adding the obtained results we have:

$$
F_{i}=0.5\left[\begin{array}{l}
\bar{F}_{1}\left(p_{1}, 0\right)+\bar{F}_{2}\left(0, p_{2}\right)+\frac{\partial \bar{F}_{1}}{\partial p_{2}} \Delta p_{2}+ \\
\frac{\partial \bar{F}_{2}}{\partial p_{1}} \Delta p_{1}+\frac{\partial^{2} \bar{F}_{1}}{\partial p_{2}^{2}} \Delta p_{2}^{2}+\frac{\partial^{2} \bar{F}_{2}}{\partial p_{1}^{2}} \Delta p_{1}^{2}+\ldots
\end{array}\right],
$$

where $\bar{F}_{1}$ and $\bar{F}_{2}$ are the values of the function in the said points $\left(p_{1}, 0\right)$ and $\left(0, p_{2}\right)$. 
The mutual influence of factors is assessed by the sum of the terms containing the partial derivatives of the function at the corresponding points.

Since the sum of the terms containing partial derivatives is not zero $\psi_{\Sigma}$. Therefore, it is reasonable to determine function $F_{i}$ in the form of a sum corrected taking into account function $\psi_{\Sigma}$ and functions $F_{q}$. Unlike the methods of V.Z. Vlasov and V.I. Ershov, which are reduced to the problem of finding the extremum of several variables using numerical iterative solution methods and the method of searching for variable parameters, for example, the steepest descent, on a grid. Functions $F_{1}, F_{2}, \ldots, F_{n}$ are presented in the form (1), (2). At the same time, we represent elementary processes as a set of even simpler processes.

An increase in the number of factors taken into account results in a significant complication of the calculation and final formulas. An attempt to take into account only the most significant factors does not lead to a significant simplification of calculations. The approach to regarding problems of this class means that the problem of finding the optimal loading parameters is solved by numerical methods of nonlinear programming. The use of numerical methods allows finding the points of local extrema and does not guarantee the determination of the global extremum. In addition, it is not possible to perform an analysis of calculations to determine certain factors for the final result. In our case, it is the hardening deformation of the flat surface of the part, for example, the working surface of the tooth of the excavator bucket.

To maximize wear resistance under conditions of shock-abrasive wear, it is necessary to minimize the mass wear $[17,18]$, which after minor transformations is reduced to the form:

$$
U=f \frac{H_{a}}{H_{m}} \cdot \frac{L \cdot b \cdot \sigma_{n}}{\sigma_{b}} \cdot \frac{(1+\delta)}{\delta},
$$

where $U$ - mass wear;

$f$ - coefficient of friction;

$H_{a}$ - abrasive hardness;

$H_{m}$ - hardness of the part material;

$\sigma_{6}-$ temporary resistance to destruction;

$L, b$ - length and width of the contact surface;

$\sigma_{n}$ - standard pressure;

$\delta$ - relative elongation of the part material;

$\beta$-empirical coefficient;

$\gamma$-density of the part material.

To reduce wear, it is necessary to reduce the value of the coefficient of friction, increase the hardness of the material, the temporary resistance to fracture and the relative elongation of the material of the part. The minimum value of the coefficient of friction is reached at a certain value $\sigma_{n}=\sigma_{n}^{0}$. Its value is largely determined by the operating conditions and the geometry of the part. The relation has no extremum by $\sigma_{B}$ and $\delta$, however, if the numerator and denominator (6) are divided by $\sigma_{n}$, in the denominator we come to expression $\bar{\varepsilon} \cdot \delta$, where $\bar{\varepsilon}=\frac{\sigma_{b}}{\sigma_{n}}$ . Since $\sigma_{b}>\sigma_{n}$, then $\bar{\varepsilon}>1$. At the same time $\frac{H_{m}}{H_{a}}<1$.

Product $\bar{\varepsilon} \cdot \frac{H_{m}}{H_{a}}=\lambda$ is less than 1 , and the sum determined by statistical data:

$$
\lambda+\delta \approx \text { const } .
$$

In this case product $\lambda \cdot \delta$ provided (5) will be maximal when $\lambda \approx \delta$. For hardened Hadfield steel, when rubbing against granite with an interlayer of iron oxides $\lambda \approx \delta \approx 0.4$, when rubbing against manganite$\lambda \approx \delta \approx 0.7$.

To solve the optimization problem, we proceed as follows, the axis passing from the center of the cutting edge of the charge and perpendicular to it is denoted as $x_{l}$, and the axis perpendicular to it $-e$. In this case, e is an arbitrary energy parameter (specific energy, impulse or pressure). As a result of calculating the deformation process (at the first stage), dependence $e\left(x_{1}\right)$ was obtained. It characterizes the distribution of the energy parameter along coordinate $x_{1}$. This function is approximated by an exponential, power or other dependence with its subsequent linearization by dependence $e^{\prime}=A+B \bar{x}$, where $\bar{x}-$ a new logarithmic or another coordinate used for linearization. The distribution of pressure, specific impulse, energy of the placed charge over the surface of the part is described by dependence $\mu=f\left(q_{0}, D_{0}\right)$, i.e. it depends on $q_{0}$ and $D_{0}$, where $q_{0}-$ specific mass of the charge, $D_{0}$ - detonation velocity. The task is formulated as follows: find such values of parameters $q_{0}$ and $D_{0}$ at which dependence $e$ from dependence family $e^{\prime}$ suits dependence $\mu\left(q_{0}, D_{0}\right)$ in the best way.

A quadratic functional of the following form is taken as the criterion of the optimization:

$$
y_{\sigma}=\int_{0}^{x_{1}}\left(\mu-e^{\prime}\right)^{2} d x_{1}
$$

An optimization criterion for calculating shear deformations when moving a friction deforming tool is set similarly. Energy parameter dependence is presented in the form $e_{F}=A_{F}+B_{F} x_{1}$, and the located one $-\mu_{F}=f(q, D)$. The optimization criterion will be of the analogous form:

$$
y_{\tau}=\int_{0}^{x_{1}}\left(\mu_{F}-e_{F}^{\prime}\right)^{2} d x_{1}
$$

To determine the optimal values of the parameters of the two-stage deformation process, we compose the following system of equations:

$$
\frac{\partial y_{\sigma}}{\partial q}+\alpha_{11} \frac{\partial y_{\tau}}{\partial q}=0
$$




$$
\begin{aligned}
& \frac{\partial y_{\sigma}}{\partial D}+\alpha_{12} \frac{\partial y_{\tau}}{\partial D}=0 ; \\
& y(q, D)=0 .
\end{aligned}
$$

Here $\alpha_{11}, \alpha_{12}$ - Lagrange multipliers; $y(q, D)-$ the equation of coupling $q$ and $D$.

Multiplying equation (7) by $\frac{\partial y}{\partial D}$, and equation (8) by $\frac{\partial y}{\partial q}$ and subtracting equation (8) from equation (7) we obtain:

$$
\frac{\partial y}{\partial D}\left(\frac{\partial y_{\sigma}}{\partial q}+\alpha_{11} \frac{\partial y_{\tau}}{\partial q}\right)=\frac{\partial y}{\partial q}\left(\frac{\partial y_{\sigma}}{\partial D}+\alpha_{12} \frac{\partial y_{\tau}}{\partial D}\right)
$$

In this case, equations (9) and (10) are solved together.

If the linear approximation of $e^{\prime}$ is rough, we use the piecewise linear approximation of function $e\left(x_{1}\right)$ with any required precision. After calculating the specific mass of the explosive charge at the second stage of the calculation, we determine the contribution of reflected waves and other factors.

In the future, it is planned to experimentally and numerically verify the analytical results of the calculation and implement the method in a specific technological process.

In the case of using a flat explosive charge and dependence (4) to determine the energy parameters of the external loading and the deformed state of the hardened layer of the surface of the part, an analytical solution to the problem is possible using the methods presented in $[17,19]$ and the expression of integral (8) in elementary functions.

The proposed method can be included as a fundamental component of an integrated mathematical model for predicting the life cycle of a certain class of parts [20-22]. These classes of part are made by the parts obtained with the methods of sheet and die forging. The complex model includes the synthesis of mathematical models of the workpiece initial state, a mathematical model of the part manufacturing process (proposed in this study), the evolution of the service properties of the part, the accumulation of damage, etc. The considered mathematical model of the part manufacturing process assumes obtaining the results of the evolution of the properties of the processed parts with their maximum approximation to the operationally necessary ones. In the material of parts obtained using the methods of plastic deformation (shaping, hardening), there are significant changes in the physical and mechanical properties and structural state. It allows to significantly affect the resource and operational reliability of the parts obtained by using combined and multi-stage deformation processes. In addition, the question of the need to use hardening processing of parts manufactured by pressure processing is being resolved.

\section{Conclusions}

The proposed method for calculating the loading parameters of combined and multi-stage deformation processes makes it possible to simplify the calculation of the parameters of the hardening process and reduce the calculation time by methods involving the use of nonlinear programming methods.

The transformation of the wear dependence by mass enabled the determination of rational parameters of the plasticity of the part depending on the ratio of the hardness of the abrasive and the surface of the part and the ratio of the normal pressure of the abrasive and the strength of the material of the part. In conclusion, it should be noted that the considered technologies can be successfully used to strengthen the elements of railway tracks, tracked vehicles, in loading and unloading equipment.

\section{References}

1. T. Haikova, R. Puzyr, D. Savelov, V. Dragobetsky, R. Argat, R. Sivak, The Research of the Morphology and Mechanical Characteristics of Electric Bimetallic Contacts. In: Chenchevoy, V. et al. (eds.) CONFERENCE 2020, PAEP, IEEE, Kremenchuk, Ukraine, $\quad 579$ doi:10.1109/PAEP49887.2020.9240847

(2020).

2. T.V. Haikova, R.H. Puzyr, R.V. Levchenko, Experimental Studies on the Stress-Strain State under Drawing Aluminum-Copper Bimetal Parts Rectangular in Plan. Russian Journal of Non-Ferrous Metals, 61(4), 404 (2020). doi:10.3103/S1067821220040033

3. V.V. Drahobetskyi, N.N. Moroz, O.V. Trotsko, Optimization of geometric and technological parameters of the process of forming sheet parts with a rational choice of intensifying factors. Bulletin of Nat. Tech. Univ. KhPI, Ukraine, 32, 38 (2009)

4. M.M. Moroz, The general approach to optimization of technology of forming of metal sheet building materials. Municipal utilities. Scientific and technical collection. Series: technical sciences, Ukraine, 88, 59 (2009)

5. M.M. Moroz, V.V. Drahobetskyi, Logistic approach to the optimization of explosive stamping processes. Resource-saving technologies of production and pressure treatment of materials in mechanical engineering, Ukraine, 346 (2009)

6. M.M. Moroz, V.V. Drahobetskyi, Determination of the optimal difference in the lengths of the steps of multistage punches. Forging and stamping production. Metal processing by pressure, Ukraine, 5, 31 (2010)

7. M.M. Moroz, Optimization of deformation of sheet parts of a cabin of the KrAZ truck. Nauka i inowacja - 2011. Materialy VII Miedzynarodowej naukowipraktycznej konferencji, Przemysl, Poland, 16, 75 (2011) 
8. M.M. Moroz, Optimization of the process of deformation of layered workpieces during blasting stamping. Actual problems of society. All-Ukrainian scientific and technical conference of young scientists and specialists, Kremenchuk, Ukraine, 115 (2010)

9. M.V. Zagirnyak, A.A. Shapoval, E.A. Naumova, A.V. Lytvynenko, V.V. Drahobetskyi, V.M. Buhaichuk, A.I. Kulyk, N.V. Vysokolian, H.I. Lehotkin, Application of morphological analysis in the development of optimal technology for obtaining tapes from refractory materials. Bulletin of Mykhailo Ostrohradskyi KrNU, Ukraine, 6/2013(83), 64 (2013)

10. I.A. Miklashevich Micromechanics of destruction in generalized spaces (Minsk, Logvinov, 2003)

11. M.M. Moroz, B.P. Sereda, T.A. Vasylchenko, A.V. Hlebenko, V.A. Vydmych, Optimization of the load of the motor of the planetary drive of press-forging machines. Bulletin of Mykhailo Ostrohradskyi KrNU, Ukraine, 2/2011(67), 73 (2011)

12. M.M. Moroz, Optimization of the process of forming of body sheet metal parts of the cab of modifications of KrAZ vehicles. Bulletin of Mykhailo Ostrohradskyi KrNU, Ukraine, 4/2011(69), 97 (2011)

13. M.M. Moroz, V.V. Drahobetskyi, A.H. Markevych, General approaches to optimizing the technology of production of sheet blanks. Bulletin of Dnipropetrovsk Academician V. Lazar National Railway Transport University, Ukraine, 28, 186, (2009)

14. Y. Salenko, R. Puzyr, O. Shevchenko, V. Kulynych, O. Pedun, Numerical Simulation of Local Plastic Deformations of a Cylindrical Workpiece of a Steel Wheel Rim. In: Ivanov V. et al. (eds) Advances in Design, Simulation and Manufacturing III. DSMIE 2020. Lecture Notes in Mechanical Engineering. Springer, Cham, 1, 442 (2020). doi:10.1007/978-3030-50794-7_43

15. I.K. Savchenko, O.P. Chervinko, Ye. Turyk, I.A.Ryabtsev, Investigation of the thermomechanical state of cylindrical parts deposited with layers of austenitic and martensitic steels. Welding production, 8, 6 (2007)

16. D. Moloshtan, E. Naumova, I. Kuzev, V. Dragobetskii, S. Bogdanov, A life cycle of vehicle facing parts during production and operation. AllUkrainian scientific and technical conference «Modern trends in the development of mechanical engineering and transport», Ukraine, 19 (2020)

17. V.V. Lotous, Assessment of the effectiveness of combined explosive hardening technologies. Bulletin of Mykhailo Ostrohradskyi KrNU, Ukraine, 4/2013(81), 144 (2013)

18. O. Kurpe, V. Kukhar, R. Puzyr, V. Burko, E. Balalayeva, E. Klimov, Electric Motors Power Modes at Synchronization of Roughing Rolling Stands of Hot Strip Mill. In: Chenchevoy, V. et al. (eds.) CONFERENCE 2020, PAEP, IEEE, Kremenchuk, Ukraine, $510 \quad$ (2020). doi:10.1109/PAEP49887.2020.9240818

19. V.V. Lotous, M.V. Zagirnyak, V.V. Drahobetskyi, Optimization of the parameters of plastic deformation during the hardening of mining equipment parts. Modern resource-and-energysaving technologies of mining production: science and research journal, KrNU, Ukraine, 2/2013(12), 97 (2013)

20. V.I. Makhnenko Safety resource for the operation of welded joints and assemblies of modern structures (Kyiv, Scientific opinion, 2006)

21. I. K. Senchekov, I. A. Ryabtsev, Ye. Turyk, Block diagram of the procedure for calculating the stressstrain state of parts in the process of surfacing and subsequent operation. Automatic welding, Ukraine, 5-6, 138 (2015)

22. V.V. Dragobetsky, A.A. Shapoval, D.V. Mospan, V.V. Lotous, Hardening of excavator bucket teeth using explosion plastic deformation. Metallurgical and mining industry, Ukraine, 2, 38 (2015) 